\title{
FRECUENCIA DE ENFERMEDAD NODULAR TIROIDEA EN LOS PACIENTES QUE ASISTEN AL SERVICIO DE ENDOCRINOLOGÍA EN UN HOSPITAL DE SANTO DOMINGO, REPÚBLICA DOMINICANA, DURANTE EL PERÍODO FEBRERO-ABRIL DE 2016
}

\author{
Frequency of Nodular Thyroid Disease in patients attending the endocrinology service in a \\ hospital in Santo Domingo, Dominican Republic, during the period February-April 2016
}

\author{
Jacquelín Díaz*, Laura Pardilla**, Laura Jovine**, Laura Santana**, Andreína Guzmán**
}

Recibido: 2/8/2017 Aprobado: 6/9/2017

DOI: http://dx.doi.org/10.22206/cysa.2018.v2i1.pp25-31

\section{Resumen}

La Enfermedad Nodular Tiroidea (ENT) es motivo de frecuente consulta médica, por lo que debe ser evaluada con precisión para realizar un tratamiento adecuado, en el momento preciso. Esta afecta entre el 4-7\% de la población, siendo las mujeres las más propensas a presentar la enfermedad.

Determinamos la frecuencia de Enfermedad Nodular Tiroidea en los pacientes que asisten al servicio de endocrinología en un hospital de Santo Domingo, República Dominicana, durante el período febrero-abril de 2016, en un estudio de carácter retrospectivo, descriptivo y de corte transversal. Evaluamos 206 archivos médicos y sonografías de los pacientes diagnosticados con Enfermedad Nodular Tiroidea.

El 96\% de los pacientes, equivalente a 197 casos de los nódulos tiroideos, se presentan en mujeres, mientras que solo un $4 \%$, igual a 9 casos, fueron identificados en hombres. La aparición de nódulos se incrementa entre los rangos de edad de 50-60 años y disminuye significativamente al superar los 60 años de edad. Predominan los coloides y quistes simples, según el tipo histológico de los nódulos, con una aparición muy poco frecuente de carcinomas.

Palabras clave: Enfermedad Nodular Tiroidea; edad; sexo; histopatología.

* Médico-Endocrinólogo Instituto Nacional del Cáncer Rosa Emilia Tavares, INCART, República Dominicana. Email: diazjacr31@gmail.com

** Biointec.

\section{Summary}

Nodular Thyroid Disease is a reason for frequent medical consultation, so it must be evaluated accurately to perform an appropriate treatment, at the right time. This affects between $4-7 \%$ of the population, with women being more likely to have this disease.

We determined the frequency of Nodular Thyroid Disease in patients attending the endocrinology service in a hospital in Santo Domingo, Dominican Republic, during the period February-April 2016, in a retrospective, descriptive and cross-sectional study. We evaluated 206 medical records and sonograms of patients diagnosed with Nodular Thyroid Disease.

The 96\%, equivalent to 197 cases of thyroid nodules, occur in women, while only $4 \%$, equivalent to 9 cases, were identified in men. The appearance of nodules increases between the age ranges of 50-60 years, and decreases significantly after reaching 60 years of age. Colloids and simple cysts predominate according to the histological type of the nodules, with a very rare occurrence of carcinomas.

Keywords: Thyroid nodular disease; age; sex; histopathology. 


\section{Introducción}

La Enfermedad Nodular Tiroidea es frecuente motivo de consulta médica, afecta entre el $4-7 \%$ de la población, con una incidencia de $100 \mathrm{ca}$ sos/100.000 habitantes/año; en estudios ecográficos se detecta en hombres entre el $17 \%$ y el $25 \%$ de los casos; en mujeres, entre el $20 \%$ y el $45 \%$; y en estudios de autopsia hasta en un $87.7 \%$, en mujeres ${ }^{1}$. Debido al desconocimiento acerca de la frecuencia de Enfermedad Nodular Tiroidea en la República Dominicana, se propuso como objetivo de esta investigación determinar la frecuencia de Enfermedad Nodular Tiroidea en los pacientes que asisten a los servicios de endocrinología de un hospital de Santo Domingo, República Dominicana, durante el período febrero-abril de 2016. La importancia de esto radica en que la falta de información sobre esta patología causa a menudo que pase desapercibida por el paciente $y$, por ende, no sea tratada efectivamente, deteriorando su salud.

Una de las glándulas más importantes del sistema endocrino es la tiroides, que es una glándula encargada de regular el metabolismo del organismo; esta juega un papel imprescindible al momento de lograr la homeóstasis. Sin embargo, esta glándula puede presentar diversas patologías, como la Enfermedad Nodular Tiroidea (ENT), que se caracteriza por la presencia de nódulos en la glándula tiroides. Un nódulo no es más que un crecimiento anormal de tejido autónomo, distinto al parénquima de la glándula u órgano. Estos pueden ser benignos o malignos, que a su vez se subdividen según su histología ${ }^{2}$.

En función de lo aquí expresado, se puede inferir que esta enfermedad puede tener varios síntomas al momento de ser diagnosticado el nódulo sea duro, como un bocio visible, que implica un agrandamiento en la glándula tiroides, ronquera o cambio de la voz, dolor en el cuello, problemas respiratorios, especialmente al estar acostado, y dificultad para deglutir alimento. Los nódulos tiroideos se evalúan de acuerdo a tres pilares fundamentales, que son: los antecedentes médicos personales y familiares, el examen físico y los medios de investigación, que orientan hacia un diagnóstico aproximado o exacto, donde lo más importante es definir si se trata de un tumor maligno o uno benigno ${ }^{3}$.

Para determinar las características del tumor con exactitud, se utilizan distintos métodos de diagnóstico, como son la citología por aspiración con aguja fina (CAAF), reconocida también como biopsia por aspiración con aguja fina (BAAF). Esta posibilita determinar cuál tratamiento se aplicará en cada afectado, de acuerdo con el resultado ${ }^{4}$.

El informe realizado por el citólogo puede ser: insatisfactorio, benigno, atipia con resultado indeterminado, neoplasia folicular, lesiones malignas sospechosas o lesiones malignas definidas; en otras ocasiones, brinda un diagnóstico definido del tipo de tumor de que se trate. Además de la aspiración con aguja fina, se puede realizar un centellograma, donde utiliza una pequeña cantidad de una sustancia radiactiva, generalmente yodo radiactivo, para obtener una imágen de la glándula, y así se determina si el nódulo es hipocaptante, hipercaptante o eucaptante; asimismo, puede realizársele al paciente pruebas de sangre para medir la cantidad de hormona tiroidea (tiroxina o T4) y de hormona estimulante de la tiroides.

Según el resultado citológico, el paciente será remitido a cirugía o únicamente vigilado sin necesidad de tratamiento; si el estudio no es concluyente, se deberá repetir la punción ${ }^{5,8}$. Todos los nódulos que se descubra que contienen cáncer, o los que se sospecha que puedan ser cancerosos, deberían ser eliminados quirúrgicamente. La mayoría de los cánceres de tiroides son curables y rara vez causan problemas que pongan en peligro la vida. Cualquier nódulo tiroideo que no sea extraído deberá ser observado 
detalladamente, con un examen del nódulo cada 6 a 12 meses. Este seguimiento puede que requiera un examen físico hecho por el médico, una ecografía de la tiroides o ambos. En alguna ocasión el médico puede intentar reducir el tamaño del nódulo suministrando tratamiento con hormona tiroidea a dosis un poco más altas de las que su cuerpo necesita, lo que se llama tratamiento supresivo. Aunque esté en tratamiento supresivo con hormona tiroidea o no, puede ser necesario repetir la biopsia si el nódulo crece. También, aun cuando la biopsia sea benigna, se le podrá recomendar cirugía para extraer el nódulo si este sigue creciendo $^{9-12}$.

\section{Material y métodos}

Esta investigación fue de carácter retrospectivo, descriptiva, transversal, realizada a partir de datos recolectados durante el período febrero-abril de 2016, de fuentes secundarias. Los datos fueron obtenidos mediante la implementación de un formulario, en el cual se ingresaron los datos de los expedientes de pacientes que acudieron a los servicios de endocrinología de un hospital de Santo Domingo, República Dominicana, que presentaron nódulos tiroideos. Las variables de esta investigación incluyeron el sexo del paciente, el grupo etario, el tipo de nódulo tiroideo, la dimensión espacial del nódulo tiroideo, así como también la cantidad de nódulos presentados.

La población del estudio constó de 206 pacientes, siendo la muestra igual a la población, durante el período febrero-abril de 2016, tomando en cuenta que presentaran nódulos tiroideos durante el período de recolección de datos de la investigación. Este estudio está basado en información recolectada de fuentes secundarias, manteniendo en todo momento la confidencialidad de las personas que en estas figuran. Los datos obtenidos fueron analizados estadísticamente mediante el programa Microsoft Excel, versión Office 2016.

\section{Resultados:}

De acuerdo a los resultados obtenidos, en la gráfica 1 se muestra que un 96\%, es decir, 197 de los casos de nódulos tiroideos se presentan en mujeres, mientras que solo un $4 \%$, equivalente a 9 casos, son identificados en hombres.

\section{Gráfica 1: Distribución de nódulos tiroideos de acuerdo al sexo $(\mathrm{N}=206)$}

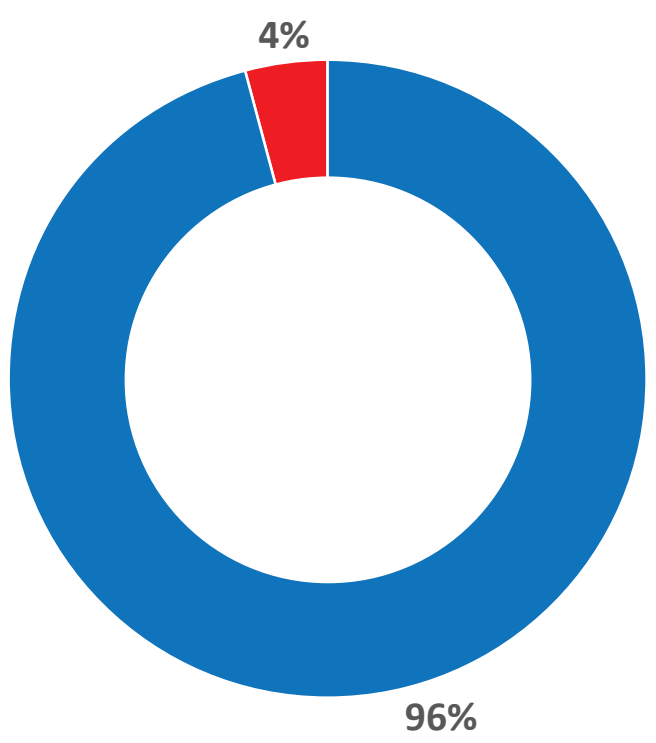

Femenido $\square$ Masculino

Fuente: Díaz J, Pardilla L, Guzmán A; Jovine L; Santana L. BioINTEC Jornada 74; abril 2017

El grupo etario con un mayor porcentaje de nódulos tiroideos es el que abarca desde los 50 a los 60 años de edad. Antes de los 40 ańos, el porcentaje va creciendo paulatinamente hasta llegar al grupo de 30 a 40 años de edad, donde aumenta significativamente; disminuyendo al llegar al grupo de 40 a 50 años de edad. A partir de los 60 años, la probabilidad de presentar nódulos tiroideos disminuye gradualmente (ver gráfica 2). 
Gráfica 2: Distribución de nódulos tiroideos según el grupo etario $(\mathrm{N}=206)$

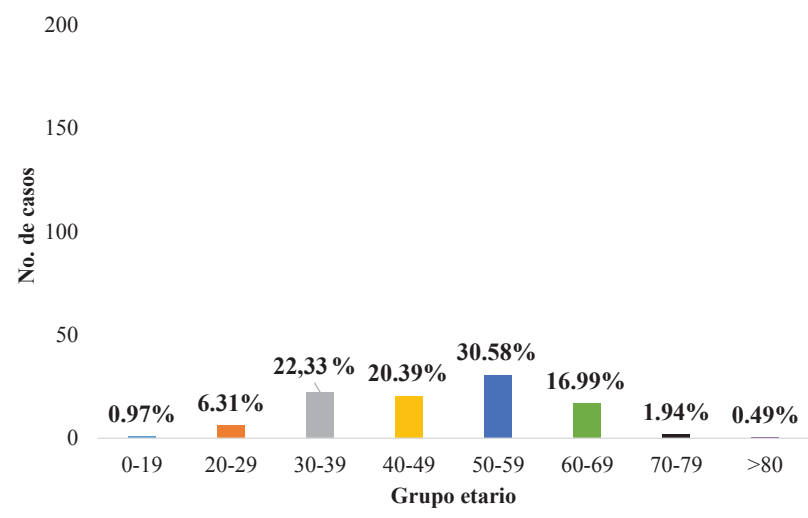

Fuente: Díaz J, Pardilla L, Guzmán A; Jovine L; Santana L. BioINTEC Jornada 74; abril 2017

La gráfica siguiente, sobre la cantidad de nódulos, muestra que lo más frecuente es la Enfermedad Nodular Tiroidea uninodular, es decir, que presenta un solo nódulo, con un $48.54 \%$, equivalente a 100 casos. La presencia de múltiples nódulos se reduce drásticamente cuando sobrepasa los dos nódulos, siendo la presencia de 8 nódulos la menos frecuente, con un porcentaje de $0.49 \%$, equivalente a un solo caso.

\section{Gráfica 3: Cantidad de nódulos tiroideos que presentaron los pacientes al momento del diag- nóstico $(\mathrm{N}=206)$}

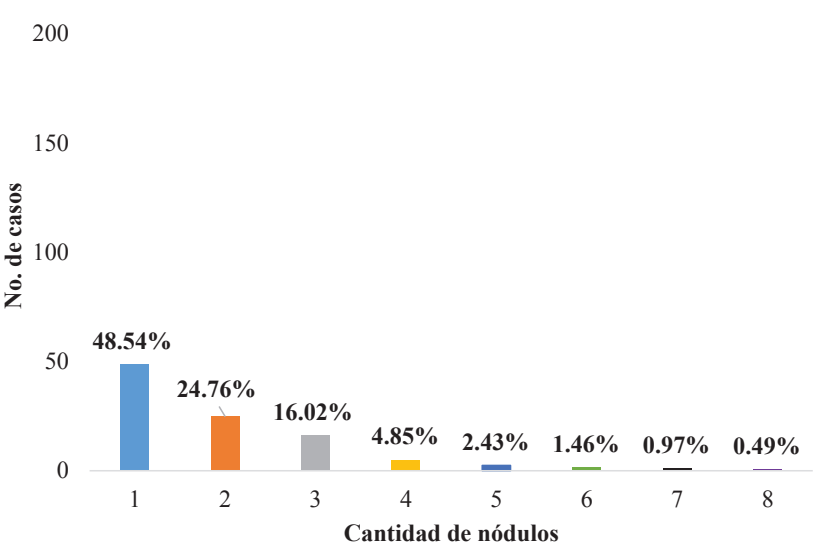

Fuente: Díaz J, Pardilla L, Guzmán A; Jovine L; Santana L. BioINTEC Jornada 74; abril 2017
De acuerdo a los resultados mostrados en la siguiente gráfica, las dimensiones espaciales más comunes son las menores de $1 \mathrm{~cm}$, siendo $0.4 \mathrm{~cm}$ la más común. La probabilidad de que un nódulo tenga las dimensiones espaciales entre $1-7 \mathrm{~cm}$ disminuye gradualmente. El rango entre $1-2 \mathrm{~cm}$ es el que presenta la segunda posición de acuerdo a su frecuencia, con un $21.36 \%$, equivalente a 44 casos. Cabe destacar que es atípico observar un nódulo con un tamaño mayor de $4 \mathrm{~cm}$ : un $1.94 \%$, equivalente a 4 casos, con una dimensión espacial de 4-5 cm; un $0.97 \%$, equivalente a 2 casos, con una dimensión espacial de 5-6 cm. La dimensión espacial entre $5-6 \mathrm{~cm}$ y $6-7 \mathrm{~cm}$ presentan la misma frecuencia, como resultado, tienen el mismo porcentaje.

Gráfica 4: Dimensiones espaciales de los nódulos tiroideos $(\mathrm{N}=206)$

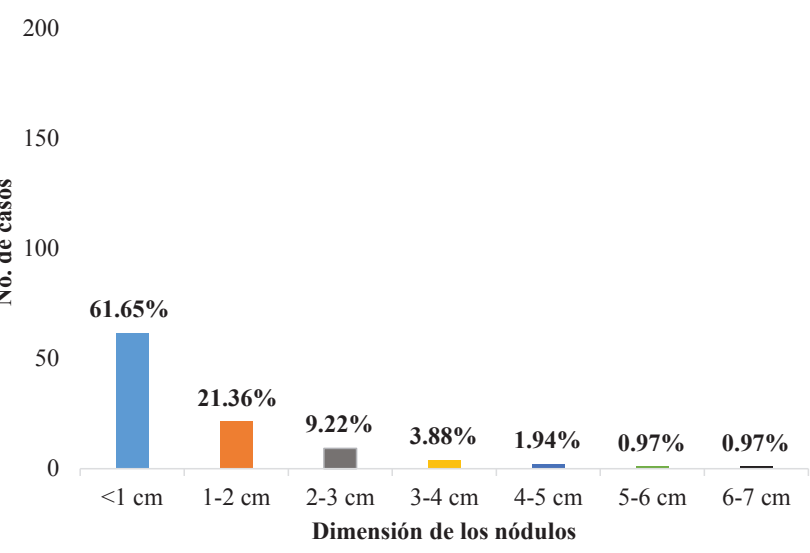

Fuente: Díaz J, Pardilla L, Guzmán A; Jovine L; Santana L. BioINTEC Jornada 74; abril 2017.

Según la histología de los nódulos diagnosticados, los quistes simples ocupan una mayor frecuencia con relación a los demás tipos de nódulos, presentando el 53.4\%, equivalente a 110 casos. Los coloides presentan una frecuencia de 16.99\%, equivalente a 35 casos, mientras que los quistes hemorrágicos ocupan un $14.08 \%$, equivalente a 29 casos. Los adenomas foliculares se encuentran en 22 casos, con un $10.68 \%$. Los linfomas tienen el tercer menor porcentaje, un $2.43 \%$, con solo 5 casos observados. Luego le sigue la tiroiditis aguda, 
presente en tres casos, con un $1.46 \%$. Del mismo grupo, la tiroiditis subaguda posee un $0.49 \%$, como el carcinoma folicular, cada uno con un solo caso (ver gráfica 5).

Gráfica 5: Recurrencia de nódulos tiroideos de acuerdo al tipo histológico diagnosticado. $(\mathrm{N}=$ 206)

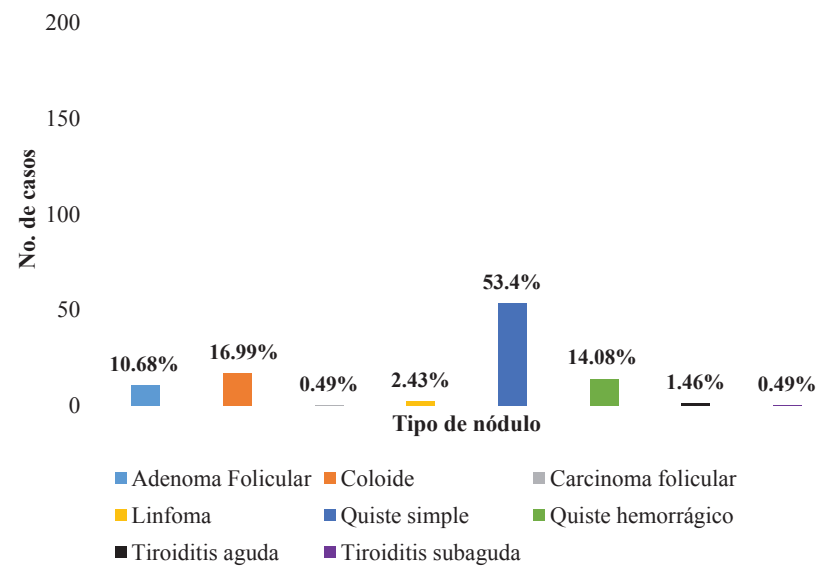

Fuente: Díaz J, Pardilla L, Guzmán A; Jovine L; Santana L. BioINTEC Jornada 74; abril 2017

\section{Discusión y conclusiones}

La frecuencia de Enfermedad Nodular Tiroidea en la pasada investigación fue de un $96 \%$, en mujeres, y solo un 4\%, en hombres. Según un estudio realizado por la doctora Naidy Rojo Quintero et al. ${ }^{1}$, en el año 2014, titulado Enfermedad nodular de tiroides, incidencia y correlación citohistológica, la frecuencia de Enfermedad Nodular Tiroidea en una población total de 104 pacientes con nódulos tiroideos reveló que 89 pacientes eran mujeres $(85,6 \%)$ y 15 eran hombres $(14,4 \%)$. Por consiguiente, los resultados de este estudio concuerdan con los de la presente investigación, pues se precisa que la frecuencia de esta enfermedad es mayor en el sexo femenino.

Por otra parte, los resultados de la constancia de la distribución de nódulos tiroideos de acuerdo al grupo etario presentan una mayor frecuencia en pacientes de 50-60 años de edad. Asimismo, los resultados muestran que la constancia de la aparición de nódulos crece gradualmente con la edad. En el estudio Enfermedad nodular de tiroides, incidencia y correlación citohistológica, referido en el párrafo precedente, el grupo de edades con mayor frecuencia de padecer esta patología fue de 40-49 años y, a su vez, el grupo de edades que presentó menor continuidad resultó de 70 años en adelante. El resultado de este estudio discrepa con los resultados de la presente investigación, pues se obtuvo una mayor frecuencia en pacientes con grupos de edades menores de 50 ańos.

La cantidad de nódulos tiroideos que presentan los pacientes de esta investigación, de una población de 206 individuos, muestra que, al momento del diagnóstico, la cantidad que presentó mayor repetición fue de un solo nódulo (51.46\%). Así, se determina que la aparición de múltiples nódulos al momento del diagnóstico es menos frecuente. El estudio titulado Comportamiento de la enfermedad nodular del tiroides en el Servicio de Cirugía General del Hospital Vladimir Iich Lenin de Holguin, realizado en Cuba por el doctor Joaquín Alejandro Solarana Ortíz ${ }^{13}$, expone que la cantidad de nódulos tiroideos más frecuente fue de un solo nódulo, por tanto, coincide con los resultados de esta investigación.

Los resultados de la evaluación de la dimensión espacial de nódulos tiroideos de la población de la presente investigación presentan una dimensión repetitiva de $0.4 \mathrm{~cm}$, y se indica que las dimensiones más frecuentes son las menores de $1 \mathrm{~cm}$. El estudio Current Diagnosis and Management of Thyroid Nodules, elaborado por los doctores Kariadi y Soetomo ${ }^{6}$ en el año 2016, indica que existe una mayor de frecuencia de nódulos con dimensiones menores a $1 \mathrm{~cm}$, puesto que generalmente la mayoría de los nódulos tiroideos son benignos, los cuales continuamente presentan este 
tipo de dimensiones.

Finalmente, de un total de 206 pacientes diagnosticados con Enfermedad Nodular Tiroidea, la histología de los nódulos diagnosticados presentó una frecuencia de $53.4 \%$ de quistes simples, siendo este tipo de nódulo el más repetitivo. A su vez, el segundo tipo de nódulo más repetitivo fueron los nódulos coloides, con un $16.99 \%$. Esto muestra que la histología de los nódulos diagnosticados en estos pacientes es mayormente de carácter benigno. El estudio Diagnostic validity of ultrasonography in thyroid nodules, del doctor Islam et al. ${ }^{14}$, en el año 2015, expone que, en una población de 101 pacientes, el 95\% de la histología de los nódulos diagnosticados fue benigna, y solo el $4.9 \%$ fue maligna.

\section{Bibliografía}

1. Rojo Quintero N, Gustavo Suárez B, Rondón Martínez E, Durruthy Willsom O, Valladares Lorenzo R. Enfermedad nodular de tiroides, incidencia y correlación citohistológica.

AMC 2016; 20(3): Mayo-Junio

2. Cooper DS, et al. American Thyroid Association Guidelines Taskforce on Thyroid N, Differentiated Thyroid C. Revised American Thyroid Association management guidelines for patients with thyroid nodules and differentiated thyroid cancer. Thyroid. 2009; 19(11): 1167-1214.

3. Anda E, Pineda J, Tonib M. Galofréb JC. Enfermedad nodular tiroidea. Science Direct. 2016; 12(13): 713-774 .

4. De Luca F, Aversa T, Alessi L, Cama V, Costanzo D, Genovese $\mathrm{C}$ et al. Thyroid nodules in childhood: Indications for biopsy and surgery. Italian Journal of Pediatrics. 2014; 40(48): $1-4$
5. Varshney R, Forest VI, Mascarella MA, Zawawi F, Rochon L, Hier MP et al. The Mcgill thyroid nodule score - does it help with indeterminate thyroid nodules? Journal of Otolaryngology. 2015; 44(1): 5.

6. Tjokorda Gde Dalem P, Kariadi, S. Current Diagnosis and Management of Thyroid Nodules. Clinical Practice. 2016; 48(11): 1-5.

7. Haugen R, Alexander EK, Keith C, Doherty GM, Mandel SJ, Nikiforov YE. American Thyroid Association Management Guidelines for Adult Patients with Thyroid Nodules and Differentiated Thyroid Cancer. American Thyroid. 2016; 26(1): 133.

8. Yoon DY, Chang SK, Choi CS et al. The prevalence and significance of incidental thyroid nodules identified on computed tomography. J Comput Assist Tomogr. 2008; 32(5): 810-815.

9. Luu MH, Fischer AH, Pisharodi L, Owens CL. Improved preoperative definitive diagnosis of papillary thyroid carcinoma in FNAs prepared with both ThinPrep and conventional smears compared with FNAs prepared with ThinPrep alone. Cancer Cytopathol. 2011; 119(1): 68-73.

10. Knox MA. Thyroid Nodules in childhood: Indications for biopsy and surgery. American Family Physician. 2013; 88 (4): 1-4.

11. Corrias A, Baronio F, Mussa A, Cassio A .Endocrinology and Diabetology (SIEDP/ ISPED): Diagnostic features of thyroid nodules in pediatrics. Arch Pediatr Adolesc Med. 2010; 164(15): 714-719.

12. López JI, Fernández de Larrinoa A, Zabala $\mathrm{R}$, Del Cura JL. El diagnóstico histológico de la patología tiroidea en biopsias guiadas por control ecográfico. Revista Española de Patología. 2009; 49(2): 97-106. 
Frecuencia de Enfermedad Nodular Tiroidea en los pacientes que asisten al servicio de endocrinología en un hospital de Santo Domingo, República Dominicana, durante el período febrero-abril de 2016

13. Solorana J, López R, Rodríguez $\mathrm{Y}$. Comportamiento de la enfermedad nodular del tiroides. SciELO.sld.cu.CCM 2013; 17 (3) : Julio-septiembre
14. Ram N, Hafeez S, Qamar S, Hussain SZ, Asghar A, Anwar Z, Islam N. Diagnostic validity of ultrasonography in thyroid nodules. Jpack Med Assoc. 2015; 65(8): 875-8. 